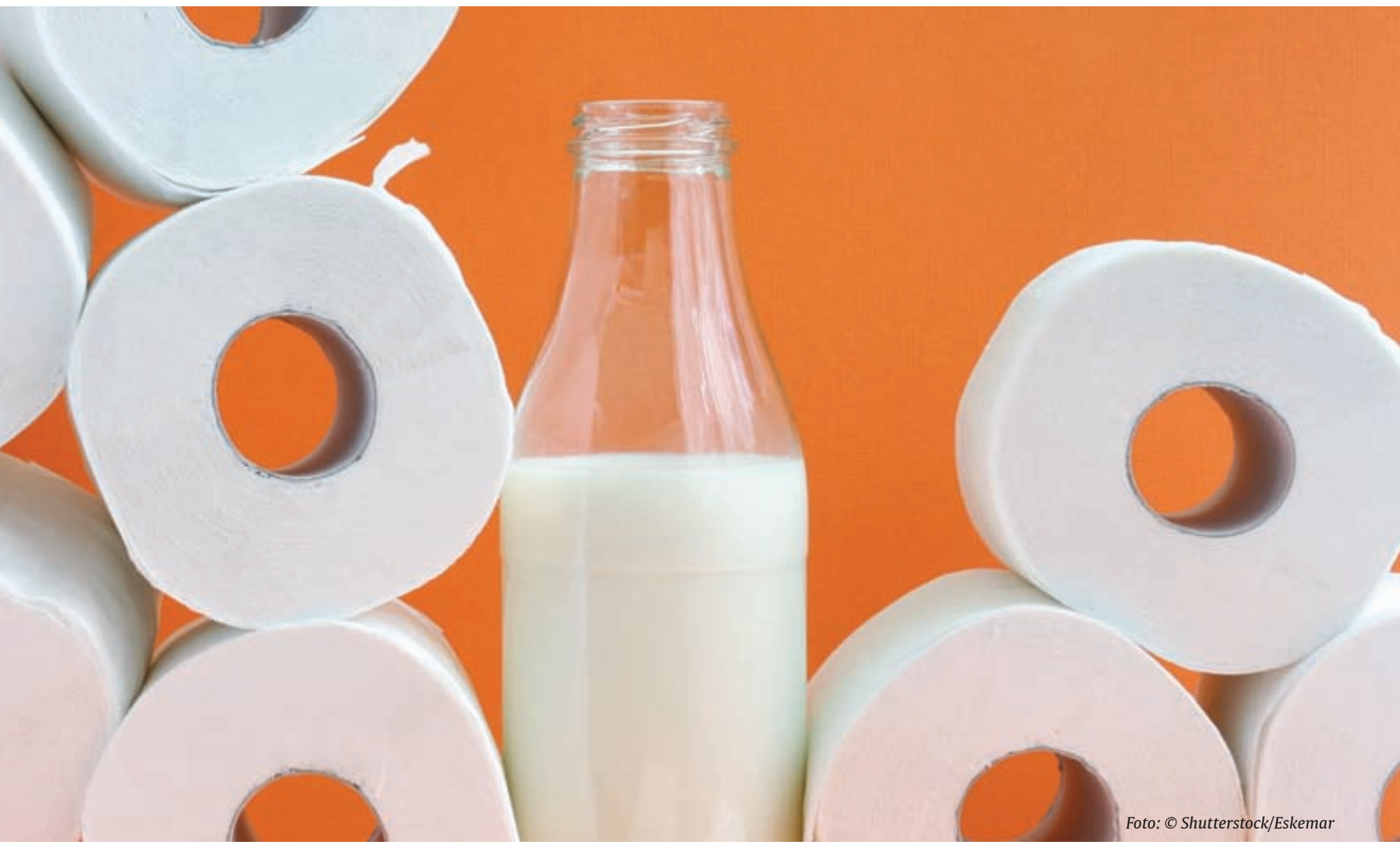

\title{
Milch wieder unbeschwert
}

\section{Symptome einer LAKTOSEINTOLERANZ können homöopathisch behandelt dauerhaft verschwinden. \\ Christa Ehrlich}

ES KOMMEN IMMER HÄUFIGER Patienten mit Verdacht auf eine Laktoseintoleranz bzw. mit einer gesicherten Diagnose dieser Erkrankung in die Praxen von Heilpraktikern. Nicht zuletzt deshalb, weil die Schulmedizin als Behandlungsoption der Milchzuckerunverträglichkeit lediglich die Gabe von Laktase kennt, dem Enzym also, das Milchzucker spaltet. Für die Dauerbehandlung eignet sich die Laktase-Gabe aber nicht. Zudem wirkt das von außen zugeführte Enzym nicht bei jedem Patienten gleich gut. Je nach im Essen enthaltener Laktosemenge wirkt es auch gar nicht. Und es ist außerdem teuer. Der Patient kommt um eine laktosefreie Ernährung also nicht herum.

Für viele Patienten ist es schwierig, sich laktosefrei zu ernähren, z. B. wenn sie berufsbedingt nicht selber kochen können und auf das Essen in der Kantine oder im Restaurant angewiesen sind. Viele Patienten wollen aber auch auf ihre Kuhmilch (statt Sojamilch) im Kaffee nicht verzichten, auch nicht auf ein Stück Schokolade hin und wieder. Also „sündigen“ sie - und bekommen Bauchschmerzen, Blähungen, Durchfall, Übelkeit, evtl. Migräne.
Es gibt einige, teilweise aus sehr unterschiedlichen homöopathischen Familien stammende Mittel, die bei Laktoseintoleranz helfen. Mitunter sind die Patienten schon nach wenigen Gaben beschwerdefrei und bleiben es, auch wenn sie in der Kantine essen, ab und an ein Stück Schokolade genießen oder einen Kaffee mit „richtiger“ Milch trinken.

Besonders häufig decken meiner Erfahrung nach folgende Mittel aus der Milch-, Magnesium- und Calciumfamilie sowohl die körperlichen als auch die psychischen Symptome der Patienten ab:

" Lac defloratum: Es ist das Milchmittel, das bei Laktoseintoleranz am häufigsten und mit den besten Erfolgen eingesetzt wird. Themen dieses Mittels sind Verlangen nach, aber auch Abneigung gegen Milch sowie die Milchunverträglichkeit. Die Beziehung zwischen Mutter und Kind, Abhängigkeit/ Unabhängigkeit, Verlassenheitsgefühl, Mangel an Selbstvertrauen sind ebenfalls Themen, die bei Menschen, die Lac defloratum benötigen, eine wichtige Rolle spielen. 


\section{Homöopathische Globuli bestehen nicht} aus Laktose, sondern aus Saccharose.

- Magnesium carbonicum und Magnesium muriaticum: Magnesiummittel sind bei der Laktoseintoleranz ebenfalls häufig indiziert, besonders Magnesium carbonicum und Magnesium muriaticum. Kennzeichnend für sie ist, dass bei Patienten, die eines dieser Mittel benötigen, die Unverträglichkeit von Milch häufig zu Krämpfen führt. Alle Patienten, die Mittel aus der Magnesiumfamilie benötigen, haben zudem eines gemeinsam: Sie sind harmoniebedürftig. Streit ertragen sie nicht, meist sind es Kinder geschiedener oder sich ständig streitender Eltern. Menschen, die Magnesium brauchen, sind oft Pazifisten, die bei aller Harmoniebedürftigkeit aber auch heftige Aggressionen entwickeln können.

- Calcium carbonicum: Mittel aus der Calciumfamilie spielen bei der Behandlung jeder Form der Milchunverträglichkeit eine große Rolle, allen voran das Calcium carbonicum. Menschen, die es benötigen, zeichnet eine starke innere Unsicherheit aus, die zu einem Festhalten an Vertrautem führt. Die Familie ist das Wichtigste, sie soll den benötigten Schutz sichern.

\section{Lac defloratum oder die Angst, den Kontakt zur Herde zu verlieren}

Eine 42 Jahre alte Frau klagt, seit etwa 1 Jahr habe sie nach Milchgenuss „qualvolle Magen-Darm-Beschwerden und Kopfschmerzen“. Von einem Arzt sei die Diagnose „Laktoseintoleranz“ bereits gestellt worden. Milch habe sie immer gerne getrunken. Die Magenschmerzen, die Darmkrämpfe und die Kopfschmerzen treten auch auf nach dem Genuss von Käse, Quark, Joghurt, Sahne, Schokolade etc.

\section{Causa/Auslösende Ursache}

Die Patientin ist Mutter von 4 Kindern im Alter zwischen 12 und 20 Jahren. Sie ist eine sehr engagierte, aber auch sehr nachgiebige Mutter. Die fortschreitende Ablösung ihrer Kinder macht ihr zu schaffen. Seit 2 Jahren ist sie wieder als Altenpflegerin tätig, die Arbeit fordert sie sehr. Seit es zunehmend Unstimmigkeiten mit Kolleginnen gibt, hat sie deutlich häufiger Beschwerden.

\section{Körperliche Symptome}

Die Patientin hat nach Milchgenuss sofort einen aufgetriebenen Bauch, krampfartige Schmerzen, Blähungen, der Bauch fühlt sich „schwer wie ein Stein“ an. Sie muss häufig sauer aufstoßen, ihr wird übel, manchmal muss sie erbrechen. Es treten zudem immer wieder pulsierende Kopfschmerzen in der Stirn und am Scheitel auf.

\section{Psychische Symptome}

Die Patientin fühlt sich überfordert, erschöpft, ausgelaugt, mit allem alleingelassen, traurig. Sie sagt: „Die Kinder waren immer das

\section{KURZ GEFASST}

1 Die

Die Schulmedizin behandelt die Laktoseintoleranz nur symptombezogen, und die Behandlung greift nicht bei allen Patienten. Auf Laktose zu verzichten ist für Betroffene unvermeidlich.

2 Homöopathische Mittel aus der Milch-, Magnesium- und Calciumfamilie haben sich in der Behandlung bewährt, mitunter auch bei Patienten, die jahrelang unter heftigen Beschwerden nach Laktoseaufnahme litten.

3 Fallbeispiele veranschaulichen die Mitteldifferenzierung und skizzieren den Behandlungsverlauf. Vorgestellt werden Lac defloratum, Magnesium carbonicum, Magnesium muriaticum und Calcium carbonicum.

Wichtigste in meinem Leben. Plötzlich wollen sie meine Fürsorge nicht mehr. Ich war ihnen gegenüber immer sehr nachgiebig. Wir hatten es so schön zusammen. Jetzt fühle ich mich in meiner Familie wie eine Fremde. Hinzu kommt, dass meine Kolleginnen, denen ich mit guten Ratschlägen helfen will, diese ablehnen und mich ausgrenzen.“

\section{Therapie und Verlauf}

Die Patientin erhält Lac defloratum C 200 (Repertorisation, s. Tab. 1, S. 26). Bereits nach der 1. Gabe treten die Kopfschmerzen nicht mehr auf, das Entfremdungsgefühl ist verschwunden. Sie kann sich besser abgrenzen, ist weniger erschöpft und traurig. Milch verträgt sie jetzt etwas besser.

Sie erhält im Abstand von einem Monat je eine Gabe Lac defloratum C 200, insgesamt 3-mal. Nach 3 Monaten sind alle körperlichen und psychischen Symptome verschwunden. Die Patientin kann wieder ohne Beschwerden Milch trinken und ihre geliebte Schokolade essen. 


\section{Magnesium carbonicum oder die Angst, im Stich gelassen zu werden}

Ein 17-Jähriger leidet unter starken Darmkrämpfen. Sie treten nach Milchgenuss, aber auch unabhängig davon auf. Besonders am Nachmittag können die Beschwerden für einige Stunden anhalten. Der Patient ist leicht reizbar, wirkt erschöpft. Ein Arzt hat bei ihm Laktoseintoleranz diagnostiziert.

\section{Causa|Auslösende Ursache}

Seit Beginn der Pubertät hat der junge Mann regelmäßig Darmkrämpfe. Er ist adoptiert, das weiß er schon, solange er zurückdenken kann. Seit einigen Jahren beschäftigt ihn die Adoption sehr. Seinen leiblichen Vater kennt er nicht. Seine leibliche Mut- ter war nicht in der Lage, ihn angemessen zu versorgen, weshalb er ihr im Alter von 3 Jahren entzogen wurde. Kontakt zur leiblichen Mutter gab es danach, allerdings verging zwischen den Kontaktaufnahmen immer viel Zeit. Seit er in der Pubertät ist, lehnt er den Kontakt zur leiblichen Mutter ab.

\section{Körperliche Symptome}

Der Patient ist groß und schlaksig, sein Gesichtsausdruck mürrisch. Er wirkt erschöpft, müde. Seine Hauptbeschwerden sind starke Darmkrämpfe und schmerzhafte Auftreibungen im Unterbauch, nachmittags sind sie am stärksten. Er hat häufig Blähungen. Nach Abgang von Winden bessern sich seine Beschwerden. Von seiner Adoptivmutter weiß er, dass er sich nach der Adopti-

\begin{tabular}{|c|c|c|c|}
\hline Repertorisation mit Synthesis & Lac defloratum & Pulsatilla & $\begin{array}{l}\text { Calcium } \\
\text { carbonicum }\end{array}$ \\
\hline Gemüt - entfremdet - Familie, von seiner & 1 & 1 & - \\
\hline Gemüt - Mutterinstinkt; übertriebener & 1 & - & 1 \\
\hline Gemüt - Nachgiebigkeit - Kindern; bei & 2 & 3 & - \\
\hline Allgemeines - Speisen und Getränke - Milch - agg. & 3 & 2 & 3 \\
\hline Allgemeines - Speisen und Getränke - Schokolade - agg. & 1 & 2 & - \\
\hline Allgemeines - Speisen und Getränke - Käse - agg. & 1 & 1 & - \\
\hline Magen - Übelkeit - Milch - agg. & 1 & 2 & 3 \\
\hline Magen - Aufstoßen - Milch, nach & 1 & - & 2 \\
\hline Magen - Erbrechen - Milch, nach & 1 & 1 & 2 \\
\hline Magen - Aufstoßen; Art des - sauer & 2 & 2 & 3 \\
\hline Magen - Übelkeit - Milch - agg. & 1 & 2 & 3 \\
\hline Abdomen - Schmerz - Milch - agg. - krampfartig & 2 & - & - \\
\hline Abdomen - Schwere - Stein; wie durch einen & 1 & 2 & - \\
\hline Kopf - Pulsieren - Stirn & 3 & 3 & 2 \\
\hline Kopf - Pulsieren - Scheitel & 3 & 1 & 1 \\
\hline \multicolumn{4}{|c|}{$\begin{array}{l}\text { Bei der Repertorisation tritt neben Calcium carbonicum und Pulsatilla Lac defloratum in den Vordergrund. Für di } \\
\text { Wahl des Mittels sind folgende Symptome entscheidend: Die heftige Reaktion auf Milch, die pulsierenden Kopf- } \\
\text { schmerzen, die Problematik der Abgrenzung gegenüber den Kindern, das Entfremdungsgefühl von der Familie. }\end{array}$} \\
\hline
\end{tabular}




\section{Weitere Leitsymptome von}

\section{Magnesium carbonicum}

- Gedeihstörungen

- „saures Kind“, d. h. alle Absonderungen riechen sauer, das Kind ist schwach und reizbar

- Kinder, die Muttermilch verweigern

- nicht gestillte Kinder

- unruhige, zappelige Beine

- krampfartige Schmerzen in Magen, Luftröhre, Uterus

- Hyperazidität

- Zahnschmerzen abends, in der Schwangerschaft

- grüner, schleimiger Stuhl

- Diarrhö

- Wadenkrämpfe

- Migräne

- Leberbeschwerden

Modalitäten: Milch, Fleisch, Wärme, Berührung und Druck verschlimmern; Bewegung an frischer Luft bessert on häufig erbrochen hat und unter Darmkoliken litt. Mit den Jahren besserten sich diese Symptome. Milch und Gemüse verschlimmern, er hat schon als Kind nur mit Widerwillen Gemüse gegessen. Er friert schnell.

Psychische Symptome

Der junge Mann ist unwillig, irgendetwas zu erzählen, alle Antworten fallen kurz aus. Er kaut an den Fingernägeln, wenn er nervös ist. Seit er in der Pubertät ist, ist er sehr unzufrieden mit sich und der Welt. Er zieht sich von seinen Adoptiveltern zurück oder sucht Streit. In der Schule ist er überfordert.

Er hat Angst, dass keiner ihn mag, wenn er so ist wie jetzt; insbesondere dass seine Adoptiveltern ihn nicht mehr mögen, sie liebt er sehr. In den ersten Jahren nach der Adoption wurde er schnell wütend, was sich mit den Jahren legte. Bis zur Pubertät hatte er ein gutes Verhältnis zu seiner Adoptivmutter. Im nächsten Jahr steht das Abitur an, die ständigen Schmerzen verhindern aber die erforderlichen Leistungen in der Schule.

\begin{tabular}{|c|c|c|c|}
\hline Repertorisation mit Synthesis & $\begin{array}{l}\text { Magnesium } \\
\text { carbonicum }\end{array}$ & $\begin{array}{l}\text { Natrium } \\
\text { muriaticum }\end{array}$ & Sulfur \\
\hline Gemüt - Beschwerden durch - Vernachlässigung; durch & 1 & 2 & 1 \\
\hline Gemüt - Verlassen zu sein; Gefühl & 2 & 2 & 1 \\
\hline Gemüt - Beißen - Nägel & 2 & 2 & 2 \\
\hline Gemüt - Furcht - Ablehnung, Zurückweisung; vor & 2 & 2 & - \\
\hline Gemüt - Antworten - Abneigung zu antworten & - & 2 & 2 \\
\hline Allgemeines - Speisen und Getränke - Milch - agg. & 2 & 2 & 3 \\
\hline Allgemeines - Speisen und Getränke - Gemüse - agg. & 2 & 1 & 2 \\
\hline Allgemeines - Speisen und Getränke - Gemüse - Abneigung & 2 & 2 & 1 \\
\hline Abdomen - Schmerz - Milch - agg. - krampfartig & 1 & - & 1 \\
\hline Abdomen - Flatulenz - nachmittags & 1 & - & - \\
\hline Abdomen - Flatus; Abgang von - amel. & 1 & 1 & - \\
\hline \multicolumn{4}{|c|}{$\begin{array}{l}\text { Natrium muriaticum und Sulfur decken viele der Symptome ab. Natrium muriaticum ist ein bekanntes „Kummer- } \\
\text { mittel“. Patienten, die es benötigen, können auch sehr reizbar sein. Sulfur ist selten das geeignete Mittel, wenn } \\
\text { der Patient leicht friert. Magnesium carbonicum ist für vernachlässigte und verlassene Kinder meist das richtige } \\
\text { Mittel und rückt daher in den Vordergrund. Die krampfartigen Schmerzen und die nachmittägliche Verschlimme- } \\
\text { rung bestätigen die Wahl. }\end{array}$} \\
\hline
\end{tabular}




\section{Therapie und Verlauf}

Der Patient erhält als Einmalgabe Magnesium carbonicum C 1000 (M) (Repertorisation, s. Tab. 2, S. 27). Bei der Folgeanamnese 4 Wochen später wirkt er ruhiger, weniger missmutig und erzählt, dass er wieder ausgeglichener sei, seit seine „Darmprobleme langsam immer besser werden“. Er isst jetzt manchmal freiwillig Gemüse, und es bereitet ihm keine Probleme. Milch verträgt er ebenfalls besser. In der Schule kann er sich gut konzentrieren, er wird die Abiturprüfung voraussichtlich bestehen.

Er erhält im Abstand von einem Monat je eine Gabe Magnesium carbonicum C 200, insgesamt 2-mal, danach weitere Gaben bei Bedarf.

In den nächsten Monaten haben wir Telefonkontakt. Wenn die Beschwerden sich verschlimmern, nimmt er Magnesium carbonicum C 200, es geht ihm „dann wieder gut.“ Nach einem Jahr treffe ich seine Mutter, und sie erzählt begeistert, dass er jetzt studiert, nicht mehr unter Darmkrämpfen leidet und wieder „richtig zugänglich“ ist.

\section{Magnesium muriaticum oder die Angst, die Versorgung zu verlieren}

Eine 22-jährige Frau kommt wegen anhaltender Diarrhö, verschlimmert durch Milch, und Schmerzen in der Lebergegend in die Praxis. Ärztliche Diagnose: Laktoseintoleranz

\section{Causa|Auslösende Ursache}

Die Patientin ist Studentin und vor Kurzem zu Hause ausgezogen. Sie war 12 Jahre alt, als ihre Eltern sich nach jahrelangen, heftigen Streitereien haben scheiden lassen. Nach der Scheidung lebte sie bei der Mutter. Das Verhältnis zwischen Mutter und Tochter ist angespannt, seit vor einem Jahr der neue Lebensgefährte der Mutter einzog. Kurz danach begannen die Durchfälle. Die Patientin hat ein starkes Harmoniebedürfnis und geht jedem Streit aus dem Weg.

\section{Körperliche Symptome}

Die Patientin hat 2- bis 5-mal täglich Stuhlgang. Der Stuhl ist wässrig, hell und „schießt plötzlich aus mir heraus“. Morgens ist die Stuhlfrequenz am höchsten. Nach dem Stuhlgang bleiben krampfartige Schmerzen im Rektum zurück. Die Lebergegend schmerzt, im Liegen auf der rechten Seite nehmen die Schmerzen zu. Die Leberwerte sind ohne Befund. Abends kommt die Patientin schlecht zur Ruhe. Sie leidet unter Wadenkrämpfen. Milch und Butter verschlimmern deutlich.

\section{Psychische Symptome}

Bei der Erstanamnese ist die Patientin sichtlich nervös. Sie sagt: „Ich habe sehr unter den Streitereien gelitten und immer zu vermitteln versucht. Mit meinem Vater verstehe ich mich jetzt ganz gut, er hat auch eine neue Lebensgefährtin, die ist o.k.“ Mit der Mutter habe sie sich anfangs auch gut verstanden, seit aber der neue Lebensgefährte eingezogen sei, habe sich das Verhältnis zur Mutter verschlechtert. Der Patientin sei das egal, aber die Mutter kümmere sich seither kaum noch um sie. „Wenn ich ihre Unterstützung brauche, hat sie keine Zeit und kein Interesse. Andere Mütter kochen und backen für ihre studierenden Kinder, wa-

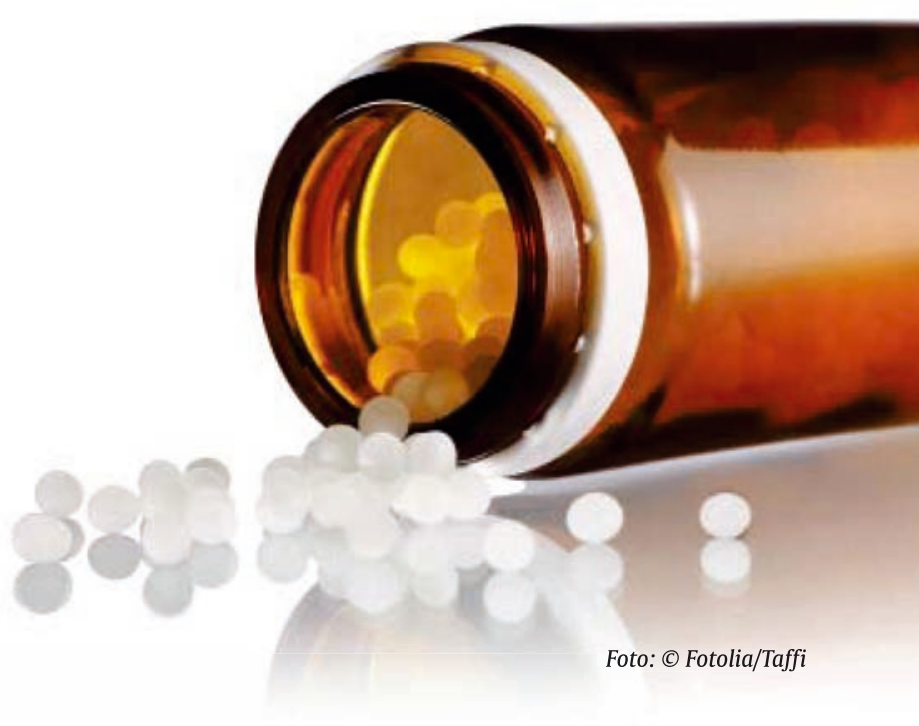

schen die Wäsche und sind immer für sie da. Meine Mutter sieht nur noch ihre neue Beziehung. Seit er eingezogen ist, habe ich diese Durchfälle. Ich weiß nicht, wie ich weiter studieren soll, wenn es so weitergeht.“

\section{Therapie und Verlauf}

Die Patientin erhält eine Gabe Magnesium muriaticum C 200 und dann im Abstand von einem Monat je eine Gabe in der Potenz C 30, insgesamt 4-mal (Repertorisation, s. Tab. 3, S. 29).

Nach der 1. Gabe kommt es zu einer Erstverschlimmerung für 2 Tage: Die Patientin hat bis zu 10-mal täglich Durchfälle. Eine Woche später setzt die Besserung der ursprünglichen Symptome ein. Die nächsten Gaben von Magnesium muriaticum erhält sie wegen der Erstverschlimmerung in der $\mathrm{C} 30$. Ab der 4. Woche nach Behandlungsbeginn hat die Patientin nur noch selten (1-mal monatlich oder nach zu viel Milch) Durchfälle. In den folgenden Monaten verschwinden auch die Leberschmerzen, die Wadenkrämpfe und die Schmerzen nach dem Stuhlgang. Mit ihrer Mutter versteht sie sich wieder besser, seit sie in einem Gespräch über ihre Bedürfnisse auf Verständnis stieß.

\section{Weitere Leitsymptome von Magnesium muriaticum \\ - ängstlich, ruhelos, verbittert, säuerlich \\ - Leber-/Galleerkrankungen (Koliken) \\ - bei Säuglingen Verstopfung und Zahnungsprobleme \\ - Unverträglichkeit von Muttermilch \\ - Kopfschmerzen \\ - Menstruationsschmerzen \\ - Wadenkrämpfe \\ - Zunge schmutzig-gelb belegt \\ Modalitäten: Baden im Meer und Salz verschlim- mern; Bewegung, Weinen, frische Luft bessern; Abneigung gegen Fisch}




\begin{tabular}{|c|c|c|c|}
\hline Repertorisation mit Synthesis & $\begin{array}{l}\text { Magnesium } \\
\text { muriaticum }\end{array}$ & $\begin{array}{l}\text { Arsenicum } \\
\text { album }\end{array}$ & Lycopodium \\
\hline $\begin{array}{l}\text { Gemüt - Beschwerden durch - Uneinigkeit, Zwietracht - } \\
\text { Eltern; zwischen den eigenen }\end{array}$ & 2 & 1 & - \\
\hline Gemüt - Streiten - Abneigung gegen & 1 & - & 1 \\
\hline Gemüt - Harmonie - Verlangen nach & 1 & - & - \\
\hline Gemüt - Ruhelosigkeit - abends - Bett - im & 3 & - & 1 \\
\hline Abdomen - Liegen - Seite; auf der - rechten; auf der - agg. & 3 & 1 & 1 \\
\hline Rektum - Diarrhö - Milch - agg. & 3 & 1 & 2 \\
\hline $\begin{array}{l}\text { Abdomen - Schmerz - Stuhlgang - nach - agg. - krampfar- } \\
\text { tig }\end{array}$ & 1 & 2 & 1 \\
\hline Stuhl - gewaltsam, plötzlich, in einem Schwall & 2 & 2 & 1 \\
\hline Extremitäten - Krämpfe - Unterschenkel - Waden - nachts & 2 & 1 & 3 \\
\hline \multicolumn{4}{|c|}{$\begin{array}{l}\text { Die Repertorisation zeigt die Nähe zu Arsenicum album und Lycopodium. Arsenicum album wäre das richtige Mit- } \\
\text { tel, wenn eine starke Ängstlichkeit vorliegen würde. Lycopodium wäre angezeigt, wenn der Machtkampf mit Vater, } \\
\text { Mutter oder dem neuen Lebensgefährten im Vordergrund stünde. Die Patientin ist jedoch, wie es für die Magnesi- } \\
\text { umfamilie typisch ist, mit viel Streit aufgewachsen, selbst harmoniebedürftig und leidet, wie es für Magnesium } \\
\text { muriaticum charakteristisch ist, unter dem Gefühl, nicht ausreichend von der Mutter versorgt zu werden. }\end{array}$} \\
\hline
\end{tabular}

\section{Calcium carbonicum oder die Angst, die schüitzende Hülle zu verlassen}

Ein 52-jähriger Mann weiß seit vielen Jahren um seine Laktoseintoleranz. Er meidet alle Milchprodukte, trotzdem reagiert er zunehmend auf geringste Mengen von Milchzucker, die in Nahrungsmitteln enthalten sind.
Körperliche Symptome

Der Patient hat starke Magenschmerzen. Als er noch Milch zu sich nahm, kam es unmittelbar nach Milchgenuss zu saurem Aufstoßen, Sodbrennen, Übelkeit und Würgen, manchmal auch zum Erbrechen. Er hat seit einigen Monaten starke Schmerzen in der rechten Schulter. Eine Verkalkung im Schultergelenk führte dazu, dass er den rechten Arm nur unter starken Schmerzen heben kann. 


\begin{tabular}{|l|l|l|l|}
\hline TABELLE 4 Calcium & & Phosphor \\
\hline Repertorisation mit Synthesis & 1 & Sulfur & 1 \\
\hline Extremitäten - Exostose & 1 & 2 \\
\hline Extremitäten - Schmerz - Schultern - Heben - Arme; der - agg. & 1 & 2 & - \\
\hline Extremitäten - Schmerz - Schultern - rechts & 3 & 2 & - \\
\hline Magen - Würgen - Milch - agg. & 2 & - & 1 \\
\hline Magen - Sodbrennen - Milch - agg. & 1 & - & 1 \\
\hline $\begin{array}{l}\text { Magen - Erbrechen - Milch, nach } \\
\text { Magen - Aufstoßens; Art des - sauer - Milch - agg. }\end{array}$ & 2 & 1 & 1 \\
\hline $\begin{array}{l}\text { Die Repertorisation stellt Sulfur und Phosphor als weitere Mittel zur Differenzierung. Die Schmerzen in der rechten } \\
\text { Schulter, die starke Reaktion auf Milch, seine Ängste um die Gesundheit und die Zurückhaltung des Patienten }\end{array}$
\end{tabular}

\section{Psychische Symptome}

Er ist zurückhaltend, deutlich übergewichtig, isst gerne und leidet unter seinem Zustand. Er hat Angst um seine Gesundheit, recherchiert im Internet jedes Symptom und sorgt sich dann sehr.

\section{Therapie und Verlauf}

Der Patient erhält 1-mal wöchentlich eine Gabe Calcium carbonicum LM 6, insgesamt 6 Monate. Nach einem Monat sind die Schulterschmerzen verschwunden, auch Magenschmerzen hat er seltener. Als Tischler ist er froh, seinen Arm wieder ohne Schmerzen bewegen zu können. Er ist immer noch eher wortkarg.

\section{Weitere Leitsymptome von Calcium carbonicum \\ - Kinder, die sich langsam entwickeln, mit Zahnungsproblemen, Polypen, Kopfschweiß \\ - phlegmatisch, schüchtern \\ - Furcht, dass etwas passieren könnte \\ - Kälteempfindlichkeit, Erkältungsneigung \\ - verzögerte Knochenheilung \\ - Drüsenvergrößerung \\ - saure Absonderungen \\ - Schwindel \\ - Schwäche oder Atemnot beim Treppensteigen \\ - Verlangen nach Eiern, unverdaulichen Dingen (Sand)}

Modalitäten: Kälte und Nässe verschlimmern; trockenes Klima bessert.
In den 6 Monaten hat er öfter Milchprodukte zu sich genommen, ohne dass es zu Symptomen kam. Er ist jedoch so daran gewöhnt, Milchprodukte zu meiden, dass er v. a. das Ausbleiben einer Reaktion auf versteckte Milchzucker als Therapieerfolg erlebt. Der Patient sorgt sich nicht mehr so stark um seine Gesundheit, die „Ängste um die Gesundheit sind ja nun nicht mehr nötig.“ "

Dieser Artikel ist online zu finden:

http://dx.doi.org/10.1055/s-0035-1549137

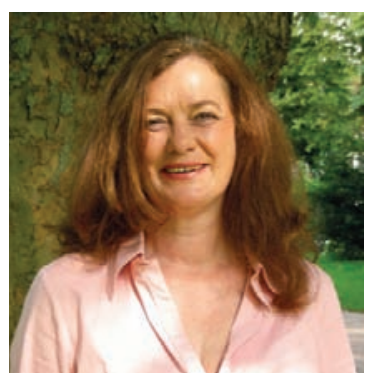

\section{HP Christa Ehrlich}

\section{Rathausstr. 11}

33602 Bielefeld

Internet: www.homoeopathie-christa-ehrlich.de

Christa Ehrlich ist Heilpraktikerin mit dem Schwerpunkt Klassische Homöopathie, SHZ-zertifiziert als Behandlerin, Dozentin und Supervisorin. Sie ist seit 1993 in Bielefeld in eigener Praxis tätig. Seit 10 Jahren ist sie Ausbildungsleiterin im Kenia-Projekt bei der Organisation „Homöopathen ohne Grenzen“. 